\title{
Truncating mutations in YIF1B cause a progressive encephalopathy with various degrees of mixed movement disorder, microcephaly, and epilepsy
}

\author{
Mohammed AlMuhaizea ${ }^{1,2} \cdot$ Rawan AIMass $^{3} \cdot$ Aljouhra AlHargan $^{3} \cdot$ Anoud AlBader $^{3} \cdot$ Eva Medico Salsench $^{4}$. \\ Jude Howaidi ${ }^{3}$. Jacie Ihinger ${ }^{5} \cdot$ Peter Karachunski $^{6} \cdot$ Amber Begtrup $^{7} \cdot$ Monica Segura Castell $^{8} \cdot$ Peter Bauer $^{8}$.

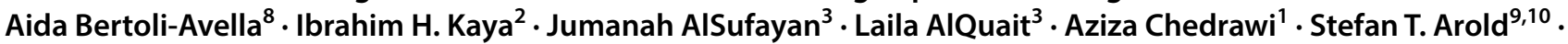 \\ Dilek Colak $^{11}$ (D) Tahsin Stefan Barakat ${ }^{4}$ (D) Namik Kaya $^{3}$ (D)
}

Received: 23 December 2019 / Revised: 21 January 2020 / Accepted: 21 January 2020

(c) Springer-Verlag GmbH Germany, part of Springer Nature 2020

Several intracellular proteins are involved in mediating vesicular transport of protein and lipid cargo from the endoplasmic reticulum (ER) to the Golgi apparatus (GA) in eukaryotic cells. Errors in membrane trafficking between ER and GA have been implicated in brain disorders [1,7], showing that these processes are critical for neuronal biogenesis. An important protein in these processes is YIF1B, an intracellular 314-residue transmembrane protein. Hippocampal neurons from Yif1B knockout (KO) mice showed that Yif1B is implicated in anterograde trafficking and Golgi architecture [1], where depletion of Yif $1 \mathrm{~b}$ caused disorganization, fragmentation, and volume reduction of the GA in pyramidal neurons.

Mohammed AlMuhaizea, and Rawan AlMass have equal contribution.

Electronic supplementary material The online version of this article (https://doi.org/10.1007/s00401-020-02128-8) contains supplementary material, which is available to authorized users.

Tahsin Stefan Barakat

t.barakat@erasmusmc.nl

Namik Kaya

nkaya@kfshrc.edu.sa

1 Department of Neurosciences, King Faisal Specialist Hospital and Research Centre (KFSHRC), Riyadh, Saudi Arabia

2 College of Medicine, AlFaisal University, Riyadh, Saudi Arabia

3 Department of Genetics, KFSHRC, Riyadh, Saudi Arabia

4 Department of Clinical Genetics, Erasmus MC University Medical Center, Rotterdam, The Netherlands

5 University of Minnesota Medical Center, Minneapolis, MN 55455, USA
Here we describe six patients from five unrelated families presenting with profound developmental and motor delay with dystonia, dysphagia, hypotonia, epilepsy and microcephaly, and homozygous truncating variants in YIFlB encountered by whole exome sequencing (WES), identifying YIFIB as a novel disease gene in humans (Fig. 1, Supplementary Fig. 1, Supplementary Table 1, online resource for detailed clinical information).

Clinical examinations revealed that all patients had an unremarkable pregnancy and birth, and no major dysmorphic features. Hypotonia and global developmental delay were noticed in infancy with smiling and partial babbling as their best achieved social and language skills. Motor development remained profoundly affected without head control, rolling or sitting. By age 2-3 years distal, limb choreiform movements started in four individuals which evolved into axial and limb dystonia with dyskinesia by the age of 4-8 years. Dystonia was unresponsive to levodopa or carbidopa but partially improved

6 Department of Neurology, University of Minnesota, Minneapolis, MN 55455, USA

7 GeneDx, Gaithersburg, MD 20877, USA

8 CENTOGENE AG, Am Strande 7, 18055 Rostock, Germany

9 Division of Biological and Environmental Sciences and Engineering (BESE), Computational Bioscience Research Center (CBRC), King Abdullah University of Science and Technology (KAUST), Thuwal 23955-6900, Saudi Arabia

10 Centre de Biochimie Structurale, CNRS, INSERM, Université de Montpellier, 34090 Montpellier, France

11 Department of Biostatistics, Epidemiology and Scientific Computing, KFSHRC, Riyadh, Saudi Arabia 
a

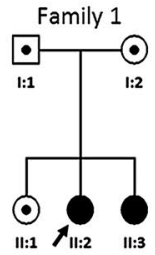

b

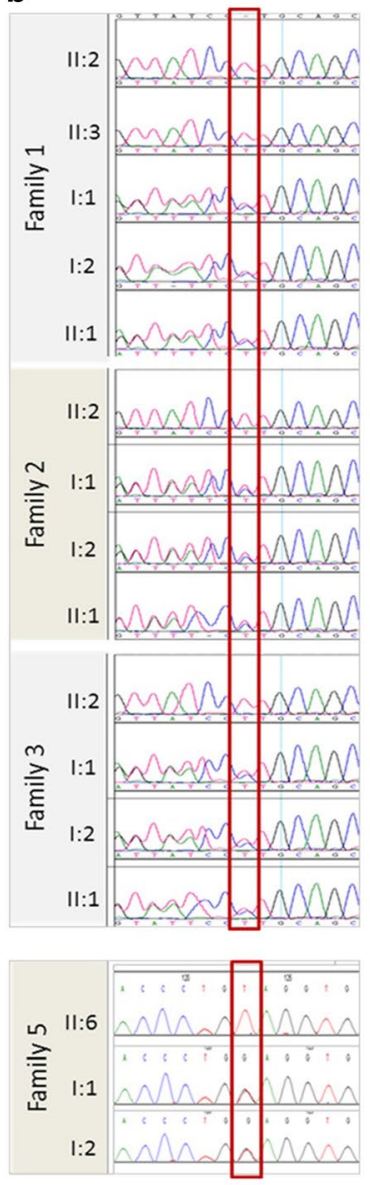

c

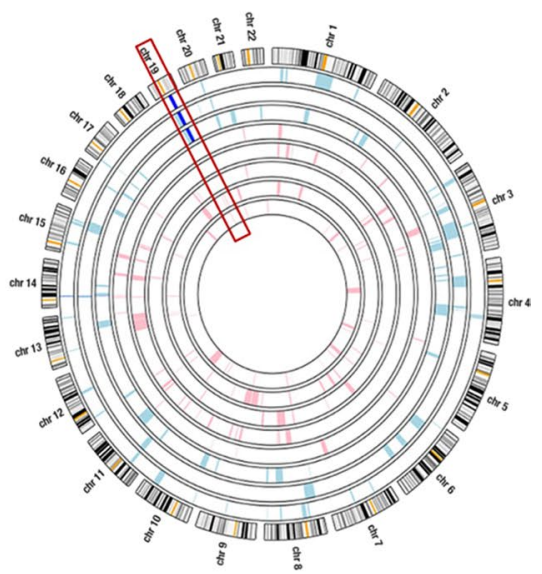

e

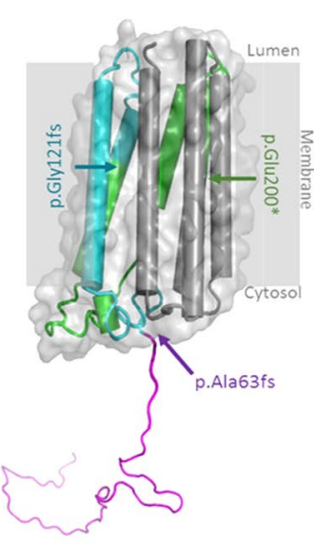

d
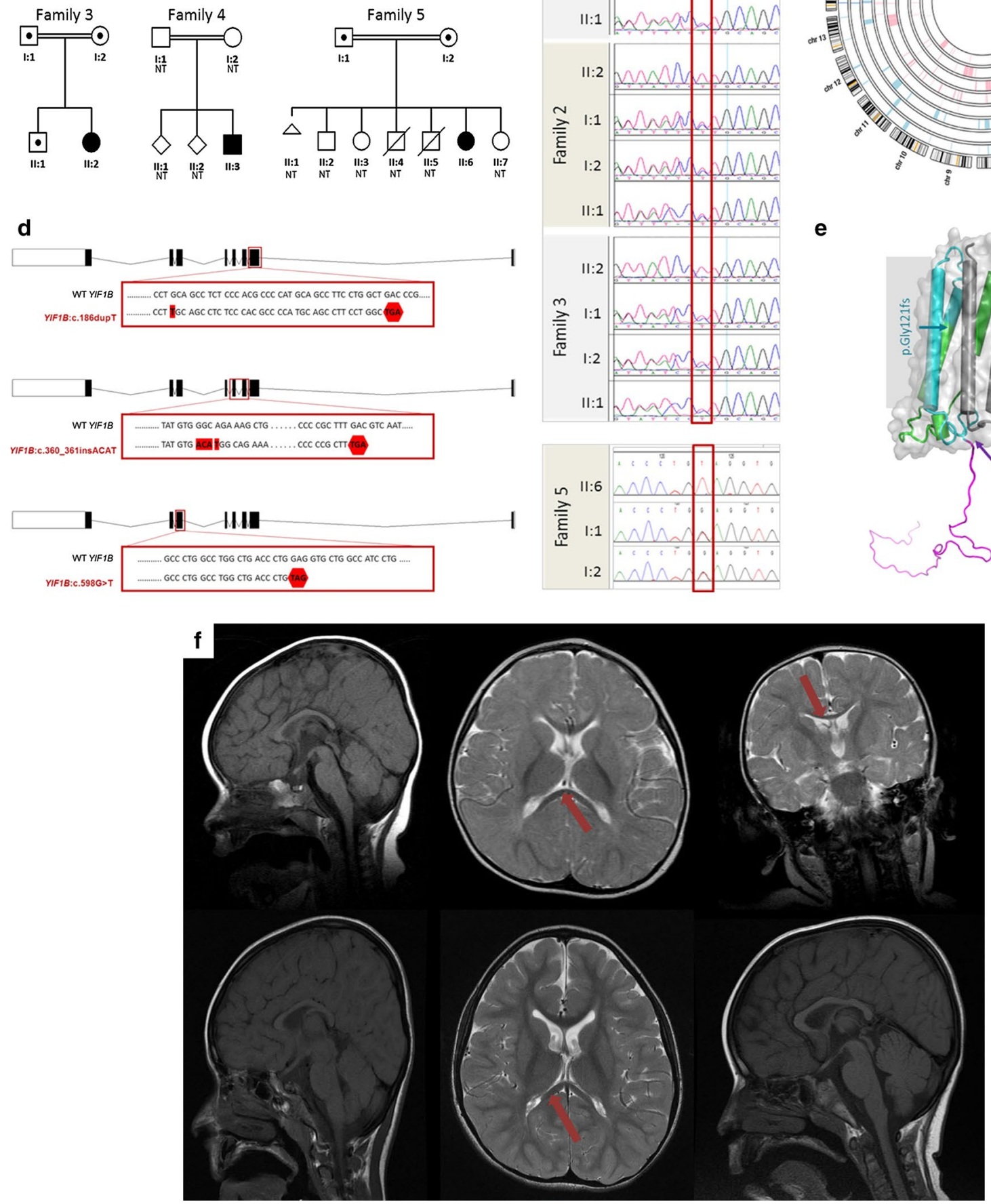

on trihexyphenidyl. Two individuals developed epileptic seizures at 6- to 8-months. EEG showed multiple bilateral epileptiform discharges and abnormal background indicating diffuse dysfunction. Microcephaly was found in all but one individual. There were no exaggerated startle, organomegaly or neurocutaneous stigmata. Hearing 
4Fig. 1 a Pedigrees of six affected individuals from five families. b Sanger sequencing reveals segregation of the variants in the tested families. c An ROH was detected on chromosome 19 including $Y I F 1 B$. d Scheme of YIF1B transcripts, affected exons, and resulting truncations. e 3D structural model for YIF1B. Helices are shown as cylinders, and the predicted molecular surface of the structured transmembrane portion of YIF1B is indicated. The flexible cytosolic region is shown in an arbitrary conformation. Protein regions retained in the variants are shown in magenta (p.Ala63fs) and magenta/cyan (p.Gly121fs) and magenta/cyan/green (p.Glu200*). The residues resulting from the frame shifts were not included (see Supplementary Figs. 2 and 3). f Brain MRI. Mild thinning of the corpus callosum was noted in all patients with the founder mutation

was normal. One case presented with cortical blindness. Repeated MRI brain imaging showed mild thinning of the corpus callosum in the majority of cases without other structural abnormalities, with brain atrophy in one case (Fig. 1f). Other standard metabolic investigations in serum and liquor were unremarkable. Hence, all individuals with truncating $Y I F 1 B$ variants shared a similar phenotypical spectrum. All affected residues encountered were highly conserved and identified variants (c.186dupT:p.Ala64fs; c.360_361insACAT:p.Gly121fs; c.598G > T:p.Glu200*) were absent from gnomAD and other databases of healthy individuals with no other homozygous loss-of-function variants found (Supplementary Fig. 1b online resource).

YIF $1 B$ is widely expressed at mRNA and protein levels in all tissues including neuronal cells, in particular raphe neurons (Supplementary Figs. 2-4, online resource). Functional and network analyses identified 171 genes that are co-regulated with YIFIB in over 46,000 human RNAseq samples $(r \geq 0.9)$, that were enriched in genes involved in neurological and development disorders, and nervous system development and function ( $p$ value $<0.01$ ) (Supplementary Fig. 5a, b and Supplementary Tables 2, 3, online resource).

In rat, the YIF1B ortholog interacts with serotonin receptor 1 (5-HT1AR) which is a Gi/Go protein-coupled receptor in dendrites of serotonin neurons of the raphe nuclei. Co-localization of Yif1B and 5-HT1AR was observed in intermediate compartment small vesicles, showing Yif1B involvement in transient intracellular trafficking and modulation of 5-HT1 AR transport to dendrites $[2,3]$. Hence, defects on Yif1B may lead to the impairment of the physiological functioning of 5-HT1AR [4]. YIF1B also interacts with the lysosomal protein TAPL in humans [5]. In HeLa cells, overexpressing truncated YIF1B reduced TAPL's lysosomal localization and colocalization with truncated YIF1B in the cis-Golgi [6]. In addition, Yif1B interacts with Rab6, a recycle trafficking protein. Therefore, alterations of this interaction may lead to aggregates' accumulation in neurons, which has been reported to cause neurodegenerative diseases [1]. These findings show that to fulfill its biological function, YIF1B interacts with different proteins and controls their trafficking. Consequently, mutations affecting protein binding sites or subcellular localization of YIF1B are expected to cause trafficking deficiencies of its interaction partners.

Computational protein modelling of YIF1B predicted that the first $\sim 68$ residues form a cytosolic disordered region and indicated the presence of transmembrane helices in the remaining 250 residues (Fig. 1e, Supplementary Fig. 6a, b, online resource). The p.Gly121fs variant leads to the insertion of 31 non-related residues which are predicted to be unstructured (Supplementary Fig. 6a-c online resource). Although we cannot rule out that this variant might retain some capacity to insert into membrane, its biological function is most likely lost. The p.Ala63fs variant leads to the introduction of 12 unrelated residues following Pro62 before the stop codon, resulting in truncation before the first helix, indicating a complete loss of function. The variant p.Glu200* has 3.5 transmembrane helices missing, which is, therefore, predicted to be non-functional.

Hence, the reported disease phenotypes are corresponding to the effects of a complete loss-of-function of YIF1B. Collectively, our data provide a demonstration of the importance of $Y I F 1 B$ in humans and argue that this gene is involved in a novel neurogenetic disorder. Further functional studies are required to unravel the precise pathogenetic mechanisms of this novel disease entity.

Acknowledgements We are grateful to the patient families for their participation. This research was conducted through intramural funds (RAC\# 2120022, 2180004, 2110006) provided by King Faisal Specialist Hospital and Research Centre (KFSHRC). We would like to thank National Plan for Science, Technology and Innovation program under King Abdulaziz City for Science and Technology (NSTIP/KACST) for supporting NK and DC. We thank the King Salman Center for Disability Research for generous funds for NK. We thank the KFSHRC Genotyping and Sequencing Core Facilities at Genetics Department, Research Advisory Council Committees, Saudi Human Genome Program and Purchasing Department (Mr. Faisal Al Otaibi) for facilitating and expediting our requests. The research by STA was supported by funding from King Abdullah University of Science and Technology (KAUST) through the Award No. FCC1/1976-25 form the Office of Sponsored Research. TSB is supported by the Netherlands Organization for Scientific Research (ZonMW Veni, Grant 91617021), a Brain \& Behavior Research Foundation NARSAD Young Investigator Grant, an Erasmus MC Fellowship 2017 and Erasmus MC Human Disease Model Award 2018.

Author contributions NK conceived and designed the experiments. RAM, AAH, JH, JAS, LAQ and AB performed experiments. DC, MS, $\mathrm{PB}, \mathrm{ABA}, \mathrm{AB}$ analyzed data. DC performed bioinformatics, pathway and network analyses. SA performed protein modeling. MAM, LAQ collected specimen. MAM handled biopsies, undertook patient care and management, collected clinical data, and delineated the patients' phenotype (F1, F2, and F3). AC helped MAM to recruit one patient. TB, MSC, PB, AB-A, JI, PK collected clinical data (F4, F5). NK, TSB, EMS, MAM, DC, JH, IHK wrote the paper. 


\section{Compliance with ethical standards}

Conflict of interest MSC, $\mathrm{PB}$ and $\mathrm{ABA}$ are employees of CENTOGENE AG. AB is an employee of GeneDx. The other authors declare no conflict of interest.

\section{References}

1. Alterio J, Masson J, Diaz J, Chachlaki K, Salman H, Areias J et al (2015) Yif1B is involved in the anterograde traffic pathway and the Golgi architecture. Traffic 16:978-993. https://doi. org/10.1111/tra.12306

2. Burnett EJ, Grant KA, Davenport AT, Hemby SE, Friedman DP (2014) The effects of chronic ethanol self-administration on hippocampal 5-HT1A receptors in monkeys. Drug Alcohol Depend 136:135-142. https://doi.org/10.1016/j.drugalcdep.2014.01.002

3. Carrel D, Masson J, Al Awabdh S, Capra CB, Lenkei Z, Hamon $\mathrm{M}$ et al (2008) Targeting of the 5-HT1A serotonin receptor to neuronal dendrites is mediated by Yif1B. J Neurosci 28:8063-8073. https://doi.org/10.1523/JNEUROSCI.4487-07.2008
4. Carrel TL, McWhorter ML, Workman E, Zhang H, Wolstencroft EC, Lorson C et al (2006) Survival motor neuron function in motor axons is independent of functions required for small nuclear ribonucleoprotein biogenesis. J Neurosci 26:11014-11022. https ://doi.org/10.1523/JNEUROSCI.1637-06.2006

5. Demirel O, Waibler Z, Kalinke U, Grunebach F, Appel S, Brossart $\mathrm{P}$ et al (2007) Identification of a lysosomal peptide transport system induced during dendritic cell development. J Biol Chem 282:37836-37843. https://doi.org/10.1074/jbc.M708139200

6. Graab P, Bock C, Weiss K, Hirth A, Koller N, Braner M et al (2019) Lysosomal targeting of the ABC transporter TAPL is determined by membrane-localized charged residues. J Biol Chem 294:7308-7323. https://doi.org/10.1074/jbc.RA118.007071

7. Horton AC, Ehlers MD (2003) Neuronal polarity and trafficking. Neuron 40:277-295. https://doi.org/10.1016/s0896 $-6273(03) 00629-9$

Publisher's Note Springer Nature remains neutral with regard to jurisdictional claims in published maps and institutional affiliations. 